\title{
Hydrogen's Antiaging and Whitening Effects on Skin
}

\author{
Hye-JinKwon ${ }^{1}$ \\ ${ }^{1}$ Professor, Department of Chemical Engineering, Soongsil Univ., Seoul, Republic of Korea \\ kwonhj0070@ssu.ac.kr ${ }^{1}$
}

Article History: Received: 11 January 2021; Accepted: 27 February 2021; Published online: 5 April 2021

\begin{abstract}
This study was conducted to confirm the suitability of hydrogen as a functional cosmetic ingredient by evaluating the anti-aging and whitening effect of hydrogen, known as an excellent antioxidant. Cosmetic hydrogen powder was prepared to investigate its inhibitory activity on elastase, collagenase, and tyrosinase, and a hydrogen pack was produced using the prepared cosmetic powder to conduct clinical trials. The results showed that hydrogen powder had excellent anti-aging activation and whitening effect. In clinical trials, skin elasticity was increased by $15 \%$, skin sagging decreased by $4.53 \%$, and skin tone improved by $4.86 \%$, compared to the control group. These results suggest that hydrogen has excellent anti-aging and whitening effect. Therefore, it is highly likely that hydrogen can be used as a functional cosmetic ingredient for wrinkle improvement and skin whitening.
\end{abstract}

Keywords: Hydrogen, antiaging, whitening, elasticity, functional cosmetic

\section{Introduction}

According to the free radical theory of aging, free radicals cause aging by damaging cells and inducing oxidative stress [1]. The human body is constantly exposed to free radicals owing to metabolism occurring in organelles, such as mitochondria and endoplasmic reticulum [2]. Among these free radicals, hydroxyl (HO·) and peroxynitrite $\left(\mathrm{NO}_{3} \cdot\right)$ radicals are the most dangerous and powerful radicals, which attack the main components of the human body, including DNA, protein, and lipids. This causes permanent damage and lead to aging[3]

Many studies have already investigated the antioxidant activity of hydrogen. It is observed that as the smallest molecule, hydrogen easily enters the mitochondria and cell nucleus to remove the free radicals. In particular, hydrogen is confirmed to exhibit antioxidant activity by specifically eliminating HO. having the highest reactivity among reactive oxygen species (ROS) [4] $\mathrm{H}_{2}$ is a tasteless and odorless gas that is neutrally charged. Thus, if the gas is administered using a hydrogen therapy machine, it can be used for therapeutic purposes such as in the form of antioxidant agents, anti-inflammatory agents, and cellular signaling molecules $[5,16]$. In sports science, hydrogen gas is used to remove oxidative stress caused by exercise by consuming hydrogen water, inhaling hydrogen gas, showering using hydrogen water, and intravenously injecting hydrogen. Moreover, $\mathrm{H}_{2}$ is used in the medical field to treat psoriasis and acute inflammatory diseases via hydrogen-water baths [6]. Although the antioxidant properties of hydrogen are widely used in various fields, only few studies have investigated their effects and use in cosmetic products.

Therefore, in this study, we evaluated the in vitro skin antiaging and whitening effects using hydrogen powder and conducted a clinical test to demonstrate the beneficial effects of hydrogen on the skin. We believe that this study willfacilitate the development of new functional cosmetics in the future.

\section{Methods}

\subsection{Sample preparation}

In the experiment, hydrogen powder was provided by Boyaz Energy Co., Ltd. (Korea). The powder was processed using ICID-registered ingredients for its use in cosmetics. The ingredients are listed in Table 1. The physical properties of the as-prepared powder were determined and used in the in vitro experiment.

For clinical tests, the sample was prepared by applying the fabricated powder to a facial mask sheet and drying it. Before using the sample for the clinical experiment, water was sprayed to generate hydrogen.

Table 1 . The ingredientsof hydrogen power

\begin{tabular}{|c|c|}
\hline ICID name & Unit(\%) \\
\hline ZnO & 20 \\
\hline Zea Mays(Corn) Starch & 70 \\
\hline Helianthus Annuus seed oil & 3 \\
\hline Citric acid & 1 \\
\hline Magnesium Sulfate & 3 \\
\hline Silica & 3 \\
\hline
\end{tabular}




\subsection{In vitro experiment}

Antiaging experiment Hydrogen powder was diluted to a final concentration of 1, 10, 100, and $1000 \mu \mathrm{g} / \mathrm{mL}$. Elastase and N-succinyl-(L-Ala)3-p-nitroanilide were dissolved in $50 \mathrm{mM}$ Tris- $\mathrm{HCl}$ buffer (pH 8.6) based on the corresponding concentrations. Further, $40 \mu \mathrm{L}$ of $2.5-\mathrm{U} / \mathrm{mL}$ elastase was added to $40-\mu \mathrm{L}$ of the samples of each concentration, followed by the addition of $80 \mu \mathrm{L}$ of $0.5-\mathrm{mg} / \mathrm{mL} \mathrm{N}$-succinyl-(L-Ala)3-p-nitroanilide. After reacting at $37^{\circ} \mathrm{C}$ for $30 \mathrm{~min}$, the absorbance was measured at $445 \mathrm{~nm}$ using a microplate reader. The elastase inhibition rate was calculated according to the following equation:

Inhibition rate (\%)

$$
=\left(1-\frac{\text { absorbance of the sample }}{\text { absorbance of the control }}\right) \times 100
$$

Hydrogen powder was diluted to a final concentration of $1,10,100$, and $1000 \mu \mathrm{g} / \mathrm{mL}$. 4Phenylazobenzyloxycarbonyl-Pro-Leu-Gly-Pro-D-Arg was dissolved in $0.1 \mathrm{M}$ Tris- $\mathrm{HCl}$ buffer (pH 7.5) containing $4 \mathrm{mM} \mathrm{CaCl}_{2}$. Further, $250 \mu \mathrm{L}$ of $0.3-\mathrm{mg} / \mathrm{mL}$ 4-phenylazobenzyloxycarbonyl-Pro-Leu-Gly-Pro-D-Arg was added to $100 \mu \mathrm{L}$ of the samples of each concentration, followed by the addition of $150 \mu \mathrm{L}$ of $0.2-\mathrm{mg} / \mathrm{mL}$ collagenase. After reacting at room temperature for $20 \mathrm{~min}, 500 \mu \mathrm{L}$ of $6 \%$ citric acid and $1.5 \mathrm{~mL}$ of ethyl acetate were added to the reaction mixture. The absorbance was measured at $320 \mathrm{~nm}$ using a microplate reader and calculated using the same equation above

Whitening experimentHydrogen powder was diluted to a final concentration of $1,10,100$, and $1000 \mu \mathrm{g} / \mathrm{mL}$. Then, $200 \mu \mathrm{L}$ of $100 \mathrm{mM}$ sodium phosphate buffer ( $\mathrm{pH}$ 6.5) and $20 \mu \mathrm{L}$ of $2000-\mathrm{U} / \mu \mathrm{L}$ mushroom tyrosinase were added to $20 \mu \mathrm{L}$ of the samples of each concentration. Further, $40 \mu \mathrm{L}$ of $1.5 \mathrm{mM}$ levodopa was added to the resulting solution, and the solution reacted at $37^{\circ} \mathrm{C}$ for $15 \mathrm{~min}$. After the reaction, the absorbance was measured at $490 \mathrm{~nm}$ using a microplate reader. The tyrosinase inhibition rate was calculated according to the following equation:

Inhibition rate (\%)

$$
=\left(1-\frac{\text { absorbance of the sample }}{\text { absorbance of the control }}\right) \times 100
$$

\subsection{Clinical trial}

After stating the purpose and contents of this study, 10 women in the age group of 40-50 years voluntarily filled the consent form and were recruited. The trial was conducted with the approval of the institutional review board. The experimental group applied the facial mask prepared in this study, and the control group used a commercially available hydrogen facial mask for 4 weeks. Measurements were performed before the test, 2 weeks after application, and 4 weeks after application. The used measuring devices were as follows: Visia-CR® (Canfiled Imaging System, USA) for whitening, F-ray (Beyoung, Korea) for skin laxity, and Ballistometer BLS780 (Dia-Stron Ltd., United Kingdom) for skin elasticity.

\subsection{Data processing}

SPSS Ver.26 (IBM, USA) was used to test all calculated data for statistical significance. The Mann-Whitney $\mathrm{U}$ method test was used to test the homogeneity between the groups $(\mathrm{p}>0.1)$. The Wilcoxon signed-rank test was used to compare the effects before and after using the masks for evaluation criteria, and the Mann-Whitney $\mathrm{U}$ method test was used to test the level of change between the groups $(\mathrm{p}<0.05)$.

\section{Results and Discussion}

\subsection{Properties of hydrogen power}

The manufactured powder is white and odorless. After storing it at $-10^{\circ} \mathrm{C}, 0^{\circ} \mathrm{C}, 25^{\circ} \mathrm{C}$, and $45^{\circ} \mathrm{C}$ for 24 hours, the powder did not show any change in coagulation, agglomeration, extraction, color, etc., confirming its stability. The results also showed that hazardous substances such as lead, arsenic, and mercury were not detected in it, confirming its safety.

\subsection{In vitro test}

Anti-aging testFigure 1 shows the results of measuring the inhibitory effect of hydrogen powder on elastase and collagenase. Elastase inhibition assay was performed at the sample concentrations of $3.50 \pm 2.04,4.89 \pm$ $1.17,13.27 \pm 1.13$, and $54.84 \pm 4.39$ at $1,10,100$, and $1000 \mu \mathrm{g} / \mathrm{mL}$.

Collagenase inhibition assay, measured at the same sample concentration, showed $2.37 \pm 1.05,7.00 \pm 1.32$, $15.65 \pm 0.84$, and $40.33 \pm 1.19$. All results showed concentration-dependent trends.Elastase is a non-specific 
enzyme that breaks down elastin, an insoluble fiber protein. It is capable of causing wrinkles by digesting collagen and elastin and destroying the network structure [7]. If IC50 of oleanolic acid used as a functional raw material for wrinkle improvement and $89.0 \mu \mathrm{g} / \mathrm{mL}$ of hydrogen powder are compared in their inhibitory activities on elastase, hydrogen powder has higher inhibitory activities [8].

Collagenase produced by fibroblasts is called MMP-1 and serves as a prototype of all interstitial collagenases. Since collagenase is an MMP-1 that acts specifically on collagen, as MMP-1 activation increases, collagen is decomposed, creating wrinkles [9]. The inhibitory activity of ascorbic acid on collagenase was $74.5 \%$ at $1000 \mu \mathrm{g} / \mathrm{mL}$, respectively, and hydrogen powder showed 50\% efficacy compared to the control group.

Whitening testFigure 2 shows the results of tyrosinase inhibition assay using hydrogen powder. Sample concentrations of $1,10,100$, and $1000 \mu \mathrm{g} / \mathrm{m} \ell$ showed an inhibition rate of $3.62 \pm 0.12,3.82 \pm 0.73,13.78 \pm 4.98$, and $61.46 \pm 5.79$, showing concentration-dependent trends. In comparison to the results from Kang [10], the inhibitory activity of hydrogen powder on tyrosinase is similar to the inhibitory activity of fermented algae and arbutin. In particular, the inhibition rate of $61.46 \pm 5.79 \%$ at $1000 \mu \mathrm{g} / \mathrm{mL}$ by hydrogen powder showed higher activity than did arbutin.

A series of oxidation processes produces melanin pigment. Hydrogen is already known as an excellent antioxidant, and it has an excellent whitening effect that can inhibit the production of melanin pigment [11].

Tyrosinase, a copper $(\mathrm{Cu})$ oxidase, is an enzyme that initiates the first step of melanogenesis. It oxidizes the tyrosine substrate through DOPA (3,4-dihydroxyphenilalanine) into dopaquinone and DOPAchrome, thereby activating the synthesis of eumelanin (black brown) and pheomelanin (red yellow) [12].

Inhibition assay of tyrosinase, a critical component of melanin production, using hydrogen powder in this study showed the effects similar to that of arbutin, the positive control group, suggesting that hydrogen powder has a high potential for use as a whitening agent.

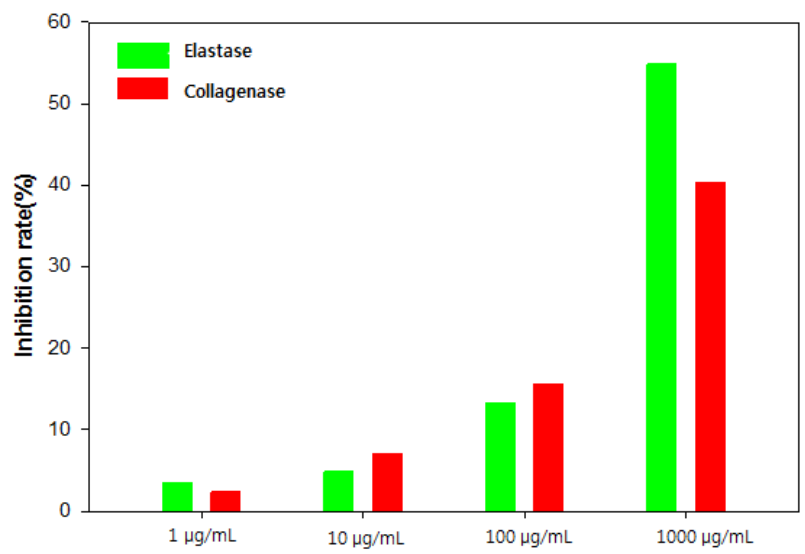

Figure2. Antiaging effects of hydrogen power

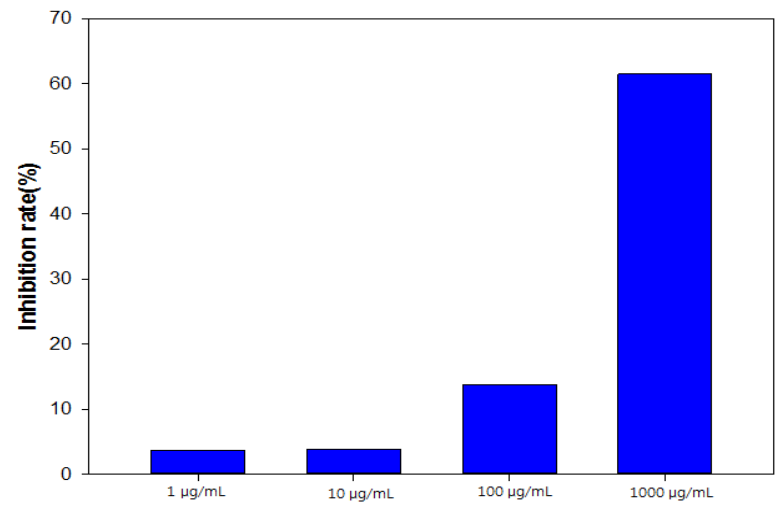

Figure2. Whitening effects of hydrogen power

3.3 Clinical trial

Prior to the experiment, a primary skin irritation test was performed on the region of the clinical subjects' 
skin that did not have pigmentation or damage. After $24 \mathrm{~h}$, the IQ Ultimate chamber was removed and the extent of skin reaction was visually assessed. Based on the Frosch \& Kligman's CTFA guidelines, skin reactivity was assessed and the skin irritation index was calculated. When the degree of skin irritation by the hydrogen mask pack was checked using the skin irritation index table generated via the Draize method, there was no anomalies found [13].

To verify the homogeneity in skin conditions prior to the use of the product between the control and experimental groups, homogeneity indexes were calculated using the results of the subjects' pre-experiment skin condition analysis. The two groups were not significantly different in terms of pre-trial skin conditions.

To improve the pigmentation of the specimen and to evaluate skin tone (brightness), the face of the subjects was photographed using Visia-CR® (Canfiled Imaging System, USA). Pigmented regions were selected in the photo of the face taken in the cross-polarized mode.

An image analysis software (Image-Pro Plus, USA) was used to determine the average V values in the pigmented regions in the photo of the face. Measurements were performed before the test and 2 and 4 weeks after the test. Skin color analysis revealed that skin pigmentation gradually faded after 2 and 4 weeks of use compared with that before use. Changes in skin color (brightness, V value) in the pigmented region were analyzed using Image-Pro Plus. The pigmented region was improved by $4.86 \%$ after 4 weeks and by $0.403 \%$ in the control group, and the difference was statistically significant.

Skin elasticity was measured using a ballistometer (BLS780; Dia-Stron Ltd., United Kingdom). This device uses the principle of vibration of the electromagnet embedded in the probe. When the device is placed on the skin surface, the form (reduction) of the vibration is detected by the control device via rapid vibration, and the value is expressed in arbitrary units. A representative measurement parameter of area (area of the elastic trajectory) was analyzed and evaluated [14]; the higher the value, the better the elasticity. In this test, the left and right cheeks were measured three times: before and after 2 and 4 weeks of use, and the average values were determined. Compared with the pre-treatment group, the skin elasticity of the cheek region in the experimental group increased by $9.40 \%$ after 2 weeks and by $15.00 \%$ after 4 weeks. On the other hand, the control group showed an increase of $2.37 \%$ after 2 weeks and of $5.36 \%$ after 4 weeks (Figure 3 ).

Skin sagging was measured using F-ray (Beyoung, Korea). This device is a three-dimensional surface measurement system (Moire topography system) using moire interference, which occurs due to the wave characteristic of light. When light is irradiated toward the subject with a grid pattern window placed in front of the subject, the periodic grid pattern and grid pattern deformed by the subject intersect with each other to create a moire-like contour line [15]. Through this contour line, the structural changes in the subject's surface can be revealed. In this test, contour images of the left and right cheek regions were taken before and after 2 and 4 weeks of use, and the angles of the contours were analyzed using an image analysis program. Compared with the pre-treatment group, the sagging of the cheek skin in the experimental group (contour angle) decreased by $2.40 \%$ after 2 weeks and by $4.53 \%$ after 4 weeks. On the other hand, in the control group, it increased by $0.12 \%$ after 2 weeks and decreased by $2.14 \%$ after 4 weeks. The rate of decrease was significantly higher in the experimental group than in the control group (Figures 4).

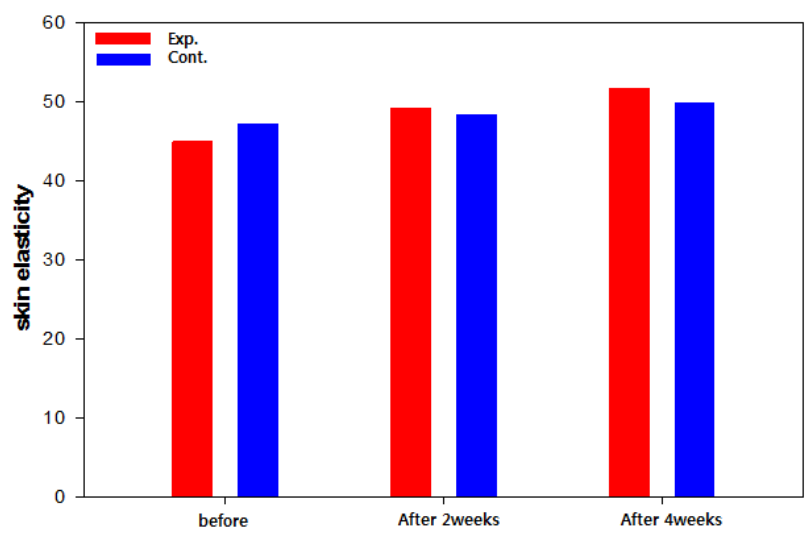

Figure 3. Skin elasticity of experimental group and control group 


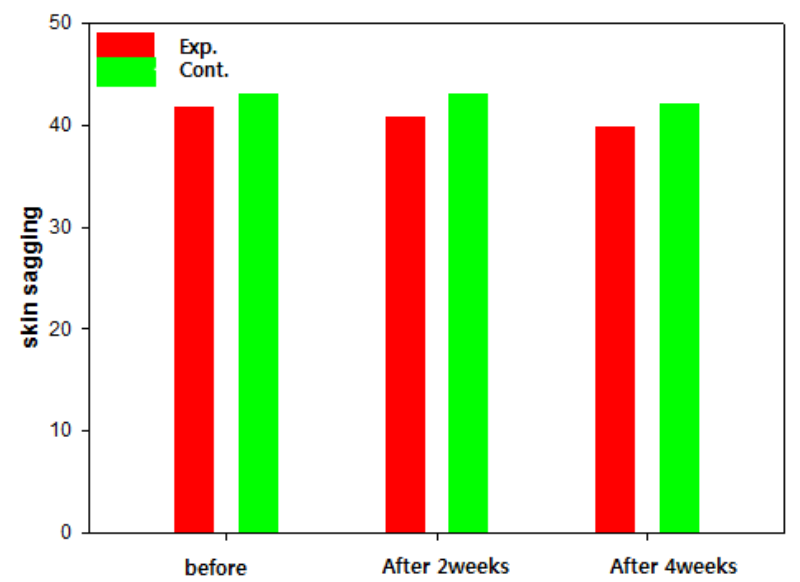

Figure 4. Skin sagging of experimental group and control group

\section{Conclusions}

This study was conducted to test the skin brightening and anti-aging effect of hydrogen powder and a hydrogen mask pack for the purpose of developing functional cosmetic materials using hydrogen.

The results showed that hydrogen has high antioxidant effects and exhibits excellent inhibitory activities on tyrosinase, elastase, and collagenase.

Therefore, hydrogen seems to be an excellent functional cosmetic material for skin brightening and antiaging. Given its diverse effects, various formulation products, in addition to the mask pack applied in this study, are highly likely to be used in the future.

\section{Acknowledgment}

6.

This research was supported by 2018 Basic Science Research Program through the National Research Foundation of Korea funded by the Education, Science and Technology. (No.2018008274)

\section{References}

1. Chinopoulos, C., Adam-Vizi, V. (2006). Calcium, mitochondria and oxidative stress in neuronal pathology. Novel aspects of an enduring theme. FEBS J. 273(3), 433-450.

2. Sauer, H., Wartenberg, M., Hescheler, J. (2001) Reactive oxygen species as intracellular messengers during cell growth and differentiation. Cell. Physiol. Biochem. 11(1), 173-186.

3. Tomizawa, S., et al. (2005). The detection and quantification of highly reactive oxygen species using the novel HPF fluorescence probe in a rat model of focal cerebral ischemia. Neurosci. Res. 53(3), 304-313.

4. Kamimura, N., Ichimiya, H., Iuchi, K., Ohta, S. (2016). Molecular hydrogen stimulates the gene expression of transcriptional coactivator pgc-1 to enhance fatty acid metabolism. Npj. Aging Mech. Dis. 2(1), 16008.

5. Ge, L., Yang, M., Yang, N. N., Yin, X. X., Song, W. G. (2017). Molecular hydrogen: A preventive and therapeutic medical gas for various diseases. Oncotarget, 8(60), 102653-102673.

6. Ohta, S. (2015). Molecular hydrogen as a novel antioxidant: Overview of the advantages of hydrogen for medical applications. Methods Enzymol. 555, 289-317.

7. Wang, C., Li, J., Liu, Q., Yang, R., Zhang, J. H., Cao, Y. P., Sun, X. J. (2011). Hydrogen-rich saline reduces oxidative stress and inflammation by inhibit of JNK and NF-kappaB activation in a rat model of amyloid-beta-induced Alzheimer's disease. Neurosci Lett, 491(2), 127-132.

8. Cai, J., Kang, Z., Liu, W. W., Luo, X., Qiang, S., Zhang, J. H., Ohta, S., Sun, X., Xu, W., Tao, H., L,i R. (2008). Hydrogen therapy reduces apoptosis in neonatal hypoxiaischemia rat model. Neurosci Lett, 441(2), 167-172.

9. Qin, Z. X., Yu, P., Qian, D. H., Song, M. B., Tan, H., Yu, Y., Li, W., Wang, H., Liu, J., Wang, Q. (2012). Hydrogen-rich saline prevents neointima formation after carotid balloon injury by suppressing ROS and the TNF-alpha/NF-kappaB pathway. Atherosclerosis, 220(2), 343-350.

10. Kang, S. W., Kim, E. J., Jung, Y. R., Ko, H. J. (2018). The Anti-Oxidant and Whitening Activities of Seaweeds Mixture Fermentation Extracts, J. Soc. Cosmet. Sci. Korea, 44(3), 327-334. 
11. Lim, J. K. (2014). A review of the usability of fucoidan extracted from brown seaweed as a functional ingredient of cosmetics, Kor. J. Aesthet. Cosmet., 12(4), 447-452.

12. Diaconeasa, Z., Leopold, L., Ruginăă, D., Ayvaz, H., Socaciu, C. (2015). Antiproliferative and antioxidant properties of anthocyanin rich extracts from blueberry and blackcurrant juice, Int. $J$. Mol. Sci., 16(2), 2352-2365.

13. Ohsawa, I., Ishikawa, M., Takahashi, K., Watanabe, M., Nishimaki, K., Yamagata, K., Katsura, K., Katayama, Y., Asoh, S., Ohta, S. (2007). Hydrogen acts as a therapeutic antioxidant by selectively reducing cytotoxic oxygen radicals. Nat Med, 13(6), 688-694.

14. Estanqueiro, M., Amaral, M. H., Sousa, Lobo, J. M. (2016). Comparison between sensory and instrumental characterization of topical formulations: Impact of thickening agents. Int. J. Cosmet. Sci., 38(4), 389-398.

15. Daudt, R. M., Back, P. I., Medeiros, Cardozo, N. L., FerreiraMarczak, L. D., Kulkamp-Guerreiro, I. C. (2015). Pinhão starch and coat extract as new natural cosmetic ingredients: Topical formulation stability and sensory analysis. Carbohydr. Polym. 134, 573-580.

16. Dunga, H., \& Mafini, C. (2019). Socio-Economic Factors Influencing The Education Of The Girl Child In Zomba, Malawi. The International Journal of Social Sciences and Humanity Studies, 11(2), 20-38. 